\title{
Optimal atrioventricular delay at rest and during exercise in patients with dual chamber pacemakers: a non-invasive assessment by continuous wave Doppler
}

\author{
DAVENDRA MEHTA, SUSAN GILMOUR, DAVID E WARD, A JOHN CAMM \\ From the Department of Cardiological Sciences, St George's Hospital Medical School, London
}

SUMMARY The optimal atrioventricular delay at rest and during exercise was investigated in nine patients with heart block and implanted dual chamber pacemakers. All patients studied had normal left ventricular function and a normal sinus node rate response to exercise. Cardiac output was measured by continuous wave Doppler and was calculated as the product of stroke distance measured by Doppler at the left ventricular outflow, aortic root area measured by $M$ mode echocardiography, and heart rate. Pacemakers were programmed in the DDD mode. Cardiac output was measured with the patient at rest while supine and while erect and at the peak of submaximal exercise (the end of stage 1 of the Bruce protocol) with the pacemakers programmed to the following atrioventricular intervals: $75-80 \mathrm{~ms}, 100-110 \mathrm{~ms}, 140-150 \mathrm{~ms}$, and $200 \mathrm{~ms}$. During exercise the basic pacing rate was programmed to 70 beats $/ \mathrm{min}$. Cardiac output at rest while supine and erect was greatest with an atrioventricular delay of 140-150 ms and it was significantly higher than that with an atrioventricular delay of $75-80 \mathrm{~ms}$. On average there was a $31 \%$ decrease in cardiac output when patients stood up. During treadmill exercise, however, cardiac output was greatest when the atrioventricular delay was $75-80 \mathrm{~ms}$, and this was significantly higher than the cardiac output with atrioventricular delays of 150 and $200 \mathrm{~ms}$. During exercise 1:1 atrioventricular relations were maintained in all patients at all atrioventricular intervals.

In patients with atrioventricular sequential pacemakers cardiac output at rest is greatest with an atrioventricular delay of $140-150 \mathrm{~ms}$ but during exercise the optimal atrioventricular delay is shorter. Rate modulation of the atrioventricular interval may improve the haemodynamic response and possibly exercise tolerance in patients with dual chamber pacemakers.

The importance of an appropriately timed atrial contraction in maintaining optimal cardiac output is now well established in patients with normal and abnormal ventricular function. ${ }^{1-4}$ The temporal relation between atrial and ventricular systole is a determinant of ventricular performance because this interval influences both ventricular filling ${ }^{5}$ and closure of the atrioventricular valve. ${ }^{67}$ Several invasive $^{8}$ and non-invasive $e^{-12}$ studies have reported that an atrioventricular interval of $150-200 \mathrm{~ms}$ is associated with maximal cardiac output at rest. We

Requests for reprints to Dr Davendra Mehta, Department of Cardiological Sciences, St George's Hospital Medical School, Cranmer Terrace, London SW 17 ORE.

Accepted for publication 11 October 1988 evaluated the optimal atrioventricular delay on exercise in patients with normal left ventricular function. Cardiac output was measured non-invasively by continuous wave Doppler techniques.

\section{Patients and methods}

Thirteen patients with dual chamber pacemakers and complete heart block were considered for the study. All had normal left ventricular function (mean ejection fraction $63.8(7.9) \%$ ) and no evidence of valvar heart disease on $\mathbf{M}$ mode echocardiography. To assess their suitability for inclusion patients underwent a three minute treadmill exercise test (treadmill speed $=2 \cdot 7 \mathrm{~km} / \mathrm{h}$ and gradient $=10 \%$ ). Doppler signals from the aorta were recorded at rest 
Table 1 Clinical and pacemaker data of the patients

\begin{tabular}{llllll}
\hline & $\begin{array}{c}\text { Age } \\
\text { Patient }(y r)\end{array}$ & Sex & Pacemaker & $\begin{array}{l}\text { Duration of } \\
\text { implantation } \\
(y r)\end{array}$ & $\begin{array}{l}\text { EF } \\
\%\end{array}$ \\
\hline 1 & 40 & M & Telectronics(Autima II) & 5 & $53 \cdot 3$ \\
2 & 58 & M & Telectronics(Autima II) & 2 & $44 \cdot 6$ \\
3 & 66 & M & Cordis(Sequicor III) & 3 & $71 \cdot 0$ \\
4 & 59 & F & Telectronics(Autima II) & 2 & $75 \cdot 5$ \\
5 & 22 & F & $\begin{array}{c}\text { Medtronic } \\
\text { (Symbios 7005C) } \ddagger\end{array}$ & 1 & $67 \cdot 9$ \\
6 & 70 & M & $\begin{array}{c}\text { Medtronic } \\
\text { (Symbios 7005C) }\end{array}$ & 1 & 62 \\
7 & 53 & M & $\begin{array}{c}\text { Telectronics(Autima II) } \\
8\end{array}$ & 1 & 59 \\
9 & 43 & M & $\begin{array}{c}\text { Medtronic } \\
\text { (Symbios 7005C) }\end{array}$ & 1 & $56 \cdot 8$ \\
\hline
\end{tabular}

*Telectronics, Sydney, Australia, +Cordis Corporation, Miami, Florida, USA, $¥$ Medtronic, Inc., Minneapolis, USA.

EF, left ventricular ejection fraction measured by $M$ mode echocardiography.

and during exercise. Four patients were excluded from the study - two with unsatisfactory Doppler signals, one with an inadequate sinus node rate response to exercise (atrial rate of $<85$ beats/minute at end of three minutes exercise) and one because 1:1 atrioventricular conduction developed on exercise. Table 1 shows selected clinical and pacemaker data of the nine patients (average age 53) included in the complete study.

\section{CARDIAC OUTPUTS MEASURED BY DOPPLER}

Cardiac output was measured by a continuous wave Doppler system (Quinton Exerdop). This "stand alone" continuous wave Doppler equipment transmits a $3 \mathrm{MHz}$ signal through a $10 \mathrm{~mm}$ diameter crystal. A proprietary circuit uses frequency analysis to process the reflected signal. The signal is sampled every $5 \mathrm{~ms}$. Only signals coming towards the transducer and at least 10 decibels above the background noise are processed. A dedicated computer calculates the flow velocity by integrating the area under the curve (systolic velocity integral) using a modified rectangular algorithm. An average of at least 10 consecutive beats was used to calculate the cardiac output at any stage of the experiment. Cardiac output was calculated as the systolic velocity integral $x$ aortic root area $\times$ heart rate. The aortic root area was calculated from the diameter of the root of the ascending aorta measured by $M$ mode echocardiography. The measurement was taken just above the level of the aortic cusps. The heart rate used for the calculation of cardiac output was averaged from 10 beats recorded simultaneously with the Doppler recordings. In previous studies cardiac output measured in this way has been shown to correlate well with measurement of cardiac output by the Fick ${ }^{13}$ and thermodilution methods ${ }^{14}$ (correlation coefficient $0 \cdot 80-0.96$ ).
The feasibility of Doppler techniques for measuring cardiac haemodynamic function during: exercise is now established..$^{1516}$ The transducer used? is small and the probe sufficiently angled so that the ultrasound signal can be easily directed parallel of nearly parallel to the blood flow in the ascending aorta. Because the transducer is small it can easily be maintained in a stable recording position during exercise and has been previously used satisfactorily at our centre to assess the haemodynamic function of post-infarction patients during exercise. ${ }^{15}$ Because $i E_{0}$ is non-invasive, this technique of measuring cardiag output is well suited for repeated assessment of patients with implanted devices.

PACEMAKER SETTINGS

During the study the pacemakers were programmed to the DDD mode. Cardiac output was measured a rest at four atrioventricular intervals with the basic $\vec{T}$ pacing rate programmed to 70 beats/minute and $90 \mathrm{D}$ beats/minute. At each rate the atrioventriculare interval was randomly programmed to $75,100,150 \frac{0}{2}$ and $200 \mathrm{~ms}$. Telectronics pacemakers have a different range of programmable atrioventricular delays - theo corresponding atrioventricular delays in patients ${ }^{\circ}$ with these pacemakers were $80,110,140$, and $200 \mathrm{~ms}$ ? For uniformity, hereafter, these are also referred to ass $75,100,150$, and $200 \mathrm{~ms}$. After each reprogramming the atrioventricular interval was confirmed byo electrocardiography.

During exercise the basic pacing rate was programmed to 70 beats/minute. At each setting of the atrioventricular interval Doppler measurements? were made five minutes after standing and at the peak. of treadmill exercises. The operator making the Doppler recordings was unaware of the pacemakero settings.

\section{EXERCISE PROTOCOL}

Patients were exercised on a treadmill for three minutes at settings identical to Bruce protocol stage 10 (gradient $=10 \%$, speed $=2 \cdot 7 \mathrm{~km} / \mathrm{h} .{ }^{17}$ Doppler measurements were made during the last $15 \mathrm{~s}$ of exercise. Faster treadmill walking speed was not usec. because it caused unacceptable background noise on Doppler recordings.

Table 2 Mean (SD) heart rate (beats/minute) at differenc stages of the protocol

\begin{tabular}{|c|c|c|c|}
\hline $\begin{array}{l}\text { Atrioventricular } \\
\text { interval }\end{array}$ & Supine & Standing & $\begin{array}{l}\text { Peak } \\
\text { exercise }\end{array}$ \\
\hline $\begin{array}{l}75 \mathrm{~ms} \\
100 \mathrm{~ms} \\
150 \mathrm{~ms} \\
200 \mathrm{~ms}\end{array}$ & $\begin{array}{l}70.8(2) \\
70.6(2) \\
70.6(2) \\
70.6(2)\end{array}$ & $\begin{array}{l}70 \cdot 9(3) \\
70 \cdot 6(2) \\
71 \cdot 2(2) \\
71 \cdot 4(3)\end{array}$ & $\begin{array}{l}102(9) \\
101.6(10) \\
101.5(9) \\
101.6(7.9)\end{array}$ \\
\hline
\end{tabular}




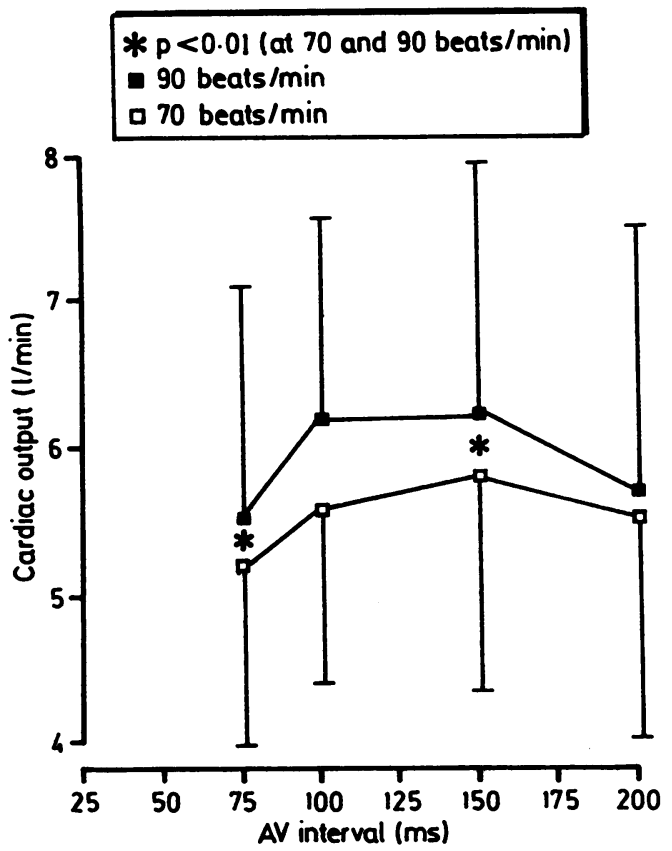

Fig 1 Comparison of mean (SD) cardiac output at the four atrioventricular delays with basic pacing rates programmed at 70 and 90 beats/minutes in resting supine patients.

Cardiac output was significantly higher at an $A V$ interval of $150 \mathrm{~ms}$ compared with an interval of $75 \mathrm{~ms}$. This result was true at both pacing rates.

\section{STATISTICAL METHOD}

All values were expressed as group mean (SD). Paired $t$ tests were used to measure statistical differences between heart rates and between cardiac outputs at various atrioventricular intervals. A $p$ value of $<0.05$ was regarded as significant.

\section{Results}

At rest, with the basic pacing rate programmed to 70 beats/minute, four patients were pacing in both the atrium and ventricle, while five were tracking the $P$ waves and pacing in the ventricle. Table 2 shows the mean pacing rates (SD) of the group at different stages of the protocol. The pacing rates at rest and during exercise at each of the four atrioventricular intervals were not significantly different. With the pacing rate programmed to 90 beats/minute, cardiac outputs at atrioventricular delays of $75,100,150$, and $200 \mathrm{~ms}$ were $5.55(1.5), 6.29(1.7), 6.42(1.8)$, and 5.88 (1.6) $1 /$ min respectively (fig 1 ). Cardiac output was significantly greater $(p<0.05)$ with an atrioventricular interval of $150 \mathrm{~ms}$ than with one of 75 $\mathrm{ms}^{18}$. No other difference was significant. Before exercise when the patient was supine with the basic

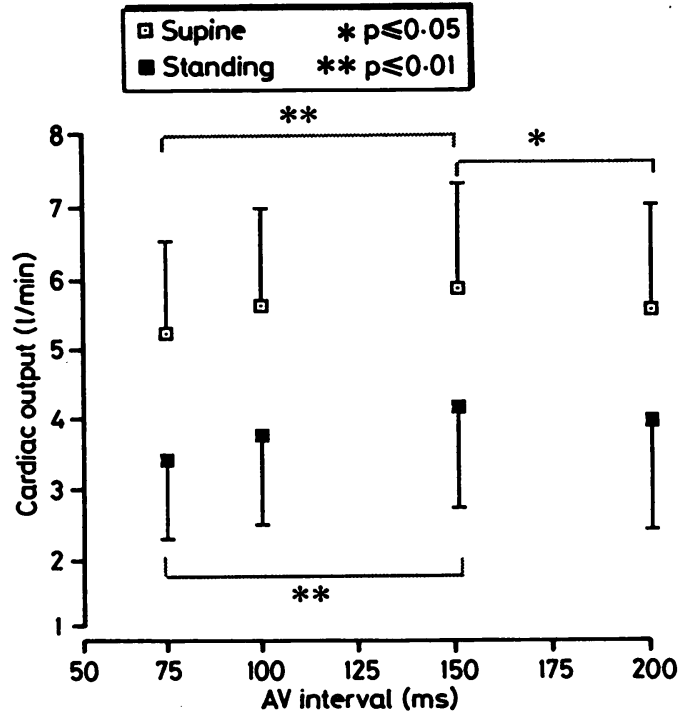

Fig 2 Changes in mean (SD) cardiac output at rest when the patient stood up. With all four atrioventricular delays there was a similar fall in cardiac output (mean $31 \%$ ).

pacing rate programmed to 70 beats/minute cardiac output was again greatest with an atrioventricular interval of $150 \mathrm{~ms}(5.81(1.5) 1 / \mathrm{min}$ ) (fig 2), and it was significantly lower at atrioventricular intervals of $75(5.20(1.3) 1 / \mathrm{min}, \mathrm{p}<0.01)$ and $200 \mathrm{~ms}(5.51$ (1.5) $1 / \mathrm{min}, \mathrm{p}<0.05)$. Whatever the atrioventricular delay, there was a $31 \%$ decrease in cardiac output on standing (fig 2). Cardiac output on standing was again greatest with an atrioventricular delay of 150 ms (4.13 (1.4) $1 / \mathrm{min})$, and this was significantly higher than the cardiac output with an atrioventricular delay of $75 \mathrm{~ms}(3.40(1.02) 1 / \mathrm{min}, \mathrm{p}<0.01)$.

The greatest cardiac output during exercise was seen with an atrioventricular delay of $75 \mathrm{~ms}(8.26$ $(1 \cdot 1) 1 / \mathrm{min})$; this was significantly higher than cardiac outputs at atrioventricular intervals of 150 and 200 $\mathrm{ms}(7.46(0.9) \mathrm{l} / \mathrm{min}(\mathrm{p}<0.05)$ and $7.85(1.1) \mathrm{l} / \mathrm{min}$ $(\mathrm{p}<0.05)$ respectively). Thus with the mild exercise used in our protocol the greatest increase in cardiac output was seen with an atrioventricular delay of 75 ms (fig 3). These changes in cardiac output between atrioventricular intervals are unrelated to changes in pacing rate because the increase in heart rate on exercise at all four atrioventricular intervals was virtually identical (table 2).

\section{Discussion}

OPTIMAL ATRIOVENTRICULAR INTERVAL

The benefits of atrioventricular sequential pacing in 


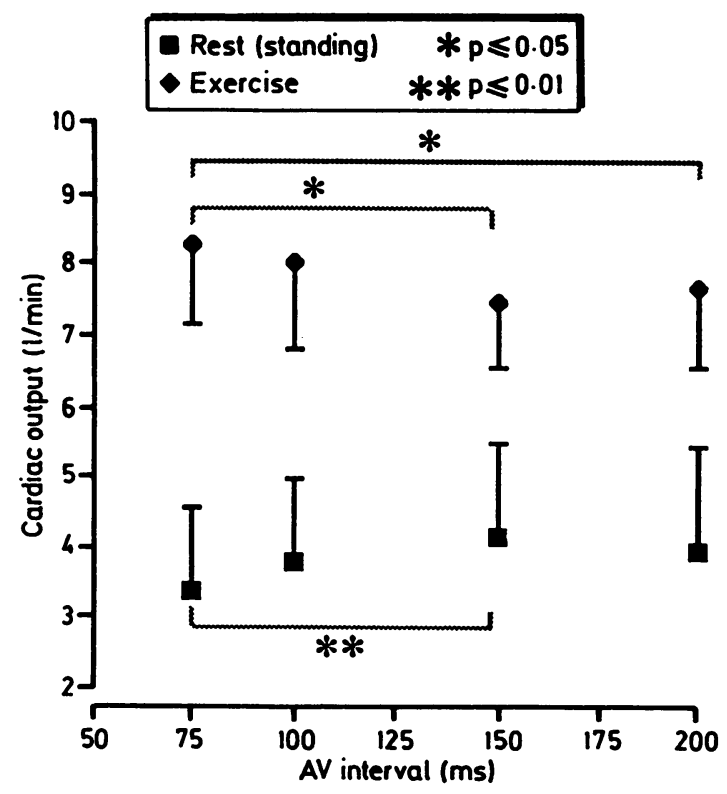

Fig 3 Mean (SD) increase in cardiac output on exercise showing an average $110 \%$ increase in cardiac output at all four atrioventricular intervals compared with values at rest (standing). The increase in cardiac output (from 3.4 (1.0) $\mathrm{l} / \mathrm{min}$ to $8 \cdot 19$ (3.1) $\mathrm{l} / \mathrm{min}$ ) was greatest with a short atrioventricular delay ( $75 \mathrm{~ms}$ ).

patients with complete heart block have been shown by invasive, ${ }^{38}$ Doppler $^{1920}$ and radionuclide ${ }^{921}$ methods. During atrioventricular sequential pacing cardiac output was significantly affected by changes in atrioventricular interval. In our patients, all of whom had normal left ventricular function, an atrioventricular interval of $150 \mathrm{~ms}$ at rest was associated with maximum cardiac output. In previous studies, however, the optimal atrioventricular delay at rest varied between $150 \mathrm{~ms}$ and $200 \mathrm{~ms}^{8-12}$ This variability may be because the results for patients with normal and abnormal left ventricular function were grouped together. Videen et al used radionuclide ventriculography to show that patients with low ejection fraction functioned better at a longer atrioventricular interval, ${ }^{21}$ whereas a shorter atrioventricular interval gave the highest cardiac output in patients with heart block complicating acute myocardial infarction. Leinbach et $a l$ and Chamberlain et al found that the optimal atrioventricular interval was $150 \mathrm{~ms}$ in patients with chronic complete heart block and $100 \mathrm{~ms}$ or less in patients with heart block complicating acute myocardial infarction. ${ }^{82}$ Hartzler et al also showed that a comparatively short atrioventricular interval was associated with a higher cardiac output during the period immediately after operation in patients in $C$. whom heart block developed at operation. ${ }^{23}$ Thus in $\vec{F}$ patients with normal left ventricular function who? are at rest an atrioventricular delay of around $150 \mathrm{~ms} \frac{\mathrm{C}}{\circ}$ is optimal. In patients with impaired left ventricular $\overline{\bar{c}}$. function a longer delay is optimal. In situations of acute stress like myocardial infarction and post- $\varrho$ operative states, where the heart rate is expected to bew faster, a relatively short atrioventricular delay was $\vec{\circ}$ found to be optimal.

Although others showed that an atrioventricular $\vec{\omega}$ delay of 150-200 ms was associated with maximal cardiac output at rest, we found that this delay is not? optimal during exercise. Our results confirm theobservations of Leman and Kartz who found that on ${ }^{\text {? }}$ exercise a shorter atrioventricular interval was $\vec{\sigma}$ associated with a larger stroke volume. ${ }^{10}$ In theiro study, however, left ventricular function was asses- ${ }_{-}-$ sed only at atrioventricular intervals of 100 and $150 \rightarrow$ ms and cardiac output was not measured. The results $\frac{\mathbb{O}}{O}$ of our study accord with the observation that exercise $\frac{C}{2}$ and its catecholamine response is associated with az shortening of the PR interval in subjects with normal $\vec{\theta}$ atrioventricular conduction. ${ }^{24}$

\section{EFFECT OF ATRIOVENTRICULAR INTERVAL ON CARDIAC EVENTS}

The benefits of atrioventricular sequential pacing are related to the timing of atrial contraction, which if appropriate leads to an increment in ventricular $\stackrel{\circ}{\rightarrow}$ filling and thereby to a ventricular contractile force $\stackrel{\vec{\partial}}{\overrightarrow{0}}$ (according to Starling's law). ${ }^{25}$ To contribute most to left ventricular filling, atrial contraction must coincide with late diastole so as to cause maximum filling of the ventricle before onset of systole. In a $\vec{\oplus}$ recent study that used Doppler assessment of mitral $\stackrel{\varrho}{\rightleftharpoons}$ flow, Iwase et al reported maximal left ventricular filling with an atrioventricular interval of $150 \mathrm{~ms}$ in .

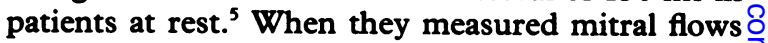
during early and late diastole they found that decreased left ventricular filling with a suboptimal $\frac{}{5}$ atrioventricular interval was mainly caused by a $>$ decreased atrial (late diastolic) component. Both very short and long atrioventricular intervals reduced left $\tilde{\sigma}$ ventricular filling.

With a physiological atrioventricular delay, atrial 0 systole causes a surge of blood into the ventricle at the $\mathrm{N}$ end of diastole. When the atrium relaxes, inertia causes this blood to continue flowing into theo ventricle, producing a region of reduced pressure $\mathbb{\Phi}$

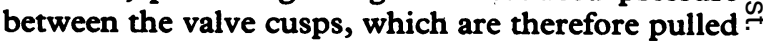
into apposition (the so called atriogenic theory of $\frac{T}{7}$ valve closure). ${ }^{2627}$ If the atrial contraction is late in diastole (as with a short atrioventricular interval), $\stackrel{\oplus}{\stackrel{\oplus}{\oplus}}$ ventricular systole starts when the atrioventricular $\mathbb{Q}$ valves are still wide open and this leads to mild mitral 
or tricuspid regurgitation. ${ }^{28}$ Furthermore, because ventricular systole starts before atrial emptying is complete the atrial contribution to left ventricular volume is less. ${ }^{7}$ Thus the cardiac output would be expected to decrease.

An atrioventricular interval that is longer than the physiological atrioventricular delay also leads to a decrease in left ventricular performance. Dean, as early as 1916, in isolated heart preparations, showed that longer intervals between atrial and ventricular systole $(>270 \mathrm{~ms})$ allowed the atrioventricular valves to move into apposition and separate again before ventricular contraction started. ${ }^{27}$ Subsequent studies have shown that an inappropriately long atrioventricular delay is associated with some mitral regurgitation at the onset of systole. ${ }^{29}{ }^{30}$ Premature atrial systole also reduces the time available for atrial filling from the pulmonary veins; thus it would be less effective than atrial systole in end diastole, when atrial filling would be maximum. Furthermore, atrial systole too early in diastole leads to premature closure of the atrioventricular valves thereby shortening the duration of left ventricular filling. ${ }^{6}$ Studies by Iwase et al did show a decreased left ventricular filling volume with long atrioventricular intervals. ${ }^{5}$

A decrease in PR interval with physiological exercise is well known. This decrease is caused by an improvement in nodal conduction under the effect of sympathetic stimulation and the decrease in vagal tone. In healthy subjects Daubert et al showed a linear shortening of the atrioventricular interval during exercise with an increase in heart rate. ${ }^{24}$ An increase in heart rate produces a disproportionate shortening of diastole compared with systole. If the atrioventricular interval were to remain constant, atrial systole would occur relatively early in diastole-a situation similar to that seen with unphysiologically long atrioventricular intervals at rest.

\section{PROBLEMS ASSOCIATED WITH UNIFORM PROGRAMMING OF ATRIOVENTRICULAR INTERVAL}

The major difficulty in programming the atrioventricular interval in patients with complete heart block and atrioventricular sequential pacemakers is the difference in the atrioventricular delay between atrioventricular sequential pacing and ventricular pacing with atrial tracking. In the case of atrioventricular pacing the atrioventricular interval begins with a paced event while with atrial tracking the atrioventricular interval begins with a sensed atrial event. Because there is a delay of $35-40 \mathrm{~ms}$ before atrial sensing, the mechanical atrioventricular delay is relatively longer despite a similar program- med atrioventricular interval. ${ }^{031}$ This makes uniform programming of the atrioventricular interval during atrial and ventricular pacing and atrial tracking difficult. Most of the present generation of dual chamber pacemakers do not distinguish between the paced and sensed atrial activity. In five of our nine patients there was tracking at the atrium at rest. This is an expected problem because of the inherent variation in sinus rate at rest from one patient to another. In previous studies, including ours, where the basic pacing rates at rest have been programmed up to 80 or 90 beats/minute so that atrioventricular pacing is established, an atrioventricular interval of 150-200 ms has still been found to be optimal. ${ }^{11} 1218$ However, during exercise in all our patients there was tracking of the atrium. This means that the relative changes in cardiac output on exercise are more relevant and indicate the benefit of a relatively short atrioventricular delay on exercise.

\section{CLINICAL IMPLICATIONS}

In patients with complete heart block and atrioventricular sequential pacemakers, rate modulation of the atrioventricular delay may improve cardiac haemodynamic function. The degree of exertion used in our study was quite mild but it is likely that the haemodynamic advantage of a shorter atrioventricular interval would also be seen with moderate to severe exertion. Further studies are needed to assess the effect on the exercise capacity of rate modulation of the atrioventricular interval.

\section{References}

1 Burchell HB. A clinical appraisal of atrial transport function. Lancet 1964;i:775-9.

2 Skinner NS, Mitchell JH, Wallace AG, Sarnoff SJ. Hemodynamic effects of altering the timing of the atrial systole. Am J Physiol 1963;205:499-503.

3 Samet P, Bernstein WH, Nathan DA, Lopez A. Atrial contribution to cardiac output in complete heart block. Am J Cardiol 1965;16:1-10.

4 Kosowsky BD, Scherlag BJ, Damato AN. Reevaluation of the atrial contribution to ventricular function: study using $\mathrm{His}$ bundle pacing. Am J Cardiol 1968;21:518-24.

5 Iwase H, Sotobata I, Yokota M, et al. Evaluation by pulsed Doppler echocardiography of the atrial contribution to left ventricular filling in patients with DDD pacemakers. Am J Cardiol 1986;58:104-9.

6 von Bibra $H$, Wirtzfeld A, Hall $R$, Ulm K, Blömer $H$. Mitral valve closure and left ventricular filling time in patients with VDD pacemakers. Assessment of the onset of left ventricular systole and the end of diastole. Br Heart J 1986;55:355-63.

7 Freedman RA, Yock PG, Echt DS, Popp RL. Effect of variation in $P Q$ interval on patterns of atrioventricular valve motion and flow in patients with 
normal ventricular function. J Am Coll Cardiol 1986;7:595-602.

8 Leinbach RC, Chamberlain DA, Kastor JA, Harthorne JW, Sanders CA. A comparison of the hemodynamic effects of ventricular and sequential AV pacing in patients with heart block. Am Heart J 1969;78:502-8.

9 Nitsch J, Seiderer M, Büll U, Lüderitz B. Evaluation of left ventricular performance by radionuclide ventriculography in patients with $A V$ versus ventricular demand pacemakers. Am Heart J 1984; 107:906-11.

10 Leman RB, Kartz JM. Radionuclide evaluation of dual chamber pacing: comparison between variable atrioventricular intervals and ventricular pacing. PACE 1985;8:408-14.

11 Faerestrand S, Ohm O-J. A time-related study of the hemodynamic benefit of atrioventricular synchronous pacing evaluated by Doppler echocardiography. $P A C E$ 1985;8:838-47.

12 Haskell RJ, French WJ. Optimal AV interval in dual chamber pacemakers. $P A C E$ 1986;9:670-5.

13 Darsee JR, Walter PF, Nutter DO. Transcutaneous Doppler method of measuring cardiac output-II. Noninvasive measurement by transcutaneous Doppler aortic blood velocity integration and $\mathbf{M}$ mode echocardiography. Am J Cardiol 1980;46: 613-8.

14 Chandraratna PA, Nanna M, McKay C, et al. Determination of cardiac output by transcutaneous continuous-wave ultrasonic Doppler computer. $A m \mathrm{~J}$ Cardiol 1984;53:234-7.

15 Mehta N, Bennett D, Mannering D, Dawkins K, Ward DE. Usefulness of noninvasive Doppler measurement of ascending aortic blood velocity and acceleration in detecting impairment of left ventricular functional response to exercise three weeks after acute myocardial infarction. Am J Cardiol 1986;58:879-84.

16 Daley PJ, Sagar KB, Wann LS. Doppler echocardiographic measurement of flow velocity in the ascending aorta during supine and upright exercise. Br Heart J 1985;54:562-7.

17 Bruce RA. Exercise testing of patients with coronary heart disease. Principles and normal standards for evaluation. Ann Clin Res 1971;3:323-32.

18 Mehta D, Gilmour S, Ward DE, et al. Optimal atrioventricular intervals in patients with dual chamber pacemakers [Abstract]. PACE 1987;10:716.
19 Stewart WJ, Dicola VC, Harthorne JW, Gillam LD, C Weyman AE. Doppler ultrasound measurement of cardiac output in patients with physiologic pace- $\stackrel{\vec{\rho}}{\rightarrow}$ makers: effects of left ventricular function and retrograde VA conduction. Am J Cardiol 1984; 54:308-12.

20 Nanda NC, Bhandari A, Barold SS, Falkoff M. Doppler echocardiographic studies in sequential atrioventricular pacing. $P A C E$ 1983;6:811-4.

21 Videen JS, Huang SK, Bazgan ID, Mechling E, Patton $\vec{\circ}$ DD. Hemodynamic comparison of ventricular pacing, atrioventricular sequential pacing and atrial $\vec{\omega}$ synchronous ventricular pacing using radionuclide $\$$ ventriculography. Am J Cardiol 1986;57:1305-8.

22 Chamberlain DA, Leinbach RC, Vassaux CE, Kastor $\sigma$ JA, DeSanctis RW, Sanders CA. Sequential atrioventricular pacing in heart block complicating acute myocardial infarction. $N$ Engl $J$ Med 1970;282:577-82.

23 Hartzler GO, Maloney JD, Curtis JJ, Barnhorst DA. Hemodynamic benefits of atrioventricular sequential $\rightarrow$ pacing after cardiac surgery. Am J Cardiol $\mathbb{D}$ 1977;40:232-6.

24 Daubert C, Ritter P, Mabo P, et al. Physiological relationship between atrioventricular interval and heart rate in healthy subjects: applications to dual $\vec{c}$ chamber pacing. PACE 1986;9:1032-9.

25 Lindrer T, Tyberg JV, Chaterjee K, et al. Effects of atrial contraction to ventricular filling: atrioventricular sequential pacing shifts the Frank Starling curve upwards [Abstract]. PACE 1983;6: A-82.

26 Henderson Y, Johnson FE. Two modes of closure of the heart valves. Heart 1912;4:69-82.

27 Dean AL Jr. The movement of the mitral cusps in relation to the cardiac cycle. Am J Physiol 1916;40:206-17.

28 Carleton RA, Passovoy M, Grattinger JS. The importance of the contribution and timing of left atrial systole. Clin Sci 1966;30:151-4.

29 Skinner NS Jr, Mitchell JH, Wallace AG, Sarnoff SJ. Hemodynamic effects of altering the timing of atrial systole. Am J Physiol 1963;205:499-504.

30 Little RC. The mechanism of closure of the mitral valve: a continuing controversy. Circulation 1979; 59:615-8.

31 Furman S. Timing mechanism during dual chamber pacing [Abstract]. PACE 1983;6:A-43. 